\title{
On the non-negative non-increasing solutions of non-linear second order differential equations.
}

\author{
I. T. KIGuradse (Tbilisi) (*)
}

Summary. - The sufficient conditions for the existence and uniqueness of solution $u(t)$ of the differential equation $u^{\prime \prime}=f\left(t, u, u^{r}\right)$, are established, satisfying the condition $\lim u(t)=$ $=u_{0}, u(t) \geq 0$ and $u^{\prime}(t) \geq 0$ for $t \in(0,+\infty)$.

This paper deals with the problem of the existence and uniqueness of so. Iution of the differential equation

$$
u^{\prime \prime}=f\left(t, u, u^{\prime}\right)
$$

defined on the interval $(0,+\infty)$ and satisfying the conditions

$$
\lim _{t \rightarrow 0} u(t)=u_{0}, u(t) \geq 0, u^{\prime}(t) \leq 0 \text { for } t \in(0,+\infty)
$$

Unlike the cases, considered earlier (of. [1], [2] [4] and the references therein indicated), it is assumed below that the function $f(t, x, y)$ is, in general, unbounded with respect to $t$; that $f(t, x, y)$ satisfies, with respect to growth relative to $y$, conditions, which are more general than conditions of the Na. gumo type.

\section{\$1. The lemmas on the a priori estimations}

We first introduce the following definitions used in this paper.

Definition 1.1 Let $0<r<+\infty, 0<\beta_{1}<\beta<+\infty$ and assume that the function $\omega_{1}(t, y)$ is defined and non-negative on the domain $t \in(0, \beta)$, $y \in(-\infty, 0]$. We say that $\omega_{1}(t, y)$ belongs to the set $B_{1}\left(r ; \beta_{1}, \beta\right)$ and write

$$
\omega_{1}(t, y) \in B_{1}\left(r ; \beta_{1}, \beta\right)
$$

if we can find a function $\rho_{1}(l)$ continuous on the halfinterval $\left(0, \beta_{1}\right]$, such that

$$
p_{1}(t) \in L\left(0, \beta_{1}\right)
$$

and if for every $\underline{\beta} \in\left(0, \beta_{1}\right]$ and an arbitrary function $u(t)$, which is absolutely-

(*) Entrata in Redazione il 26 aprile 1968 . 
continuous together with $u^{\prime}(t)$ on an interval $[\beta, \beta]$ and satisfies the inequalities

$$
0 \leq u(t) \leq r, u^{\prime}(t) \leq 0 \text { for } t \in[\beta, \beta]
$$

and

$$
u^{\prime \prime}(t) \leq \omega_{\mathrm{I}}\left(t, u^{\prime}(t)\right) \text { for } t \in[\beta, \beta]
$$

we have

$$
\left|u^{\prime}(t)\right| \leq \rho_{1}(t) \text { for } t \in\left[\underline{\beta}, \beta_{1}\right]
$$

Definition 1. 2. Let $0<r<+\infty, 0<\alpha<\alpha_{1}<+\infty$ and assume that the function $\omega_{2}(t, y)$ is defined and non-negative on a domain $t \in[\alpha,+\infty)$, $y \in(-\infty, 0]$. We say that $\omega_{2}(t, y)$ belongs to the set $B_{2}\left(r ; \alpha, \alpha_{1}\right)$ and write

$$
\omega_{2}(t, y) \in B_{2}\left(r ; \alpha, \alpha_{1}\right)
$$

if we can find a function $\rho_{2}(t)$ continuous on an interval $\left[\alpha_{1},+\infty\right)$, such that for every $\bar{\alpha} \in\left[\alpha_{1},+\infty\right)$, and for an arbitrary function $u(t)$, which is absolutely-continuous together with $u^{\prime}(t)$ on the interval $[\alpha, \bar{\alpha}]$ and satisfies the inequalities

$$
0 \leq u(t) \leq r, u^{\prime}(t) \leq 0 \text { for } \quad t \in[\alpha, \bar{\alpha}]
$$

and

$$
u^{\prime \prime}(t) \geq-\omega_{2}\left(t, u^{\prime}(t)\right) \text { for } t \in\left[\alpha_{1}, \bar{a}\right]
$$

we have

$$
\left|u^{\prime}(t)\right| \leq \rho_{2}(t) \text { for } t \in\left[\alpha_{1}, \bar{\alpha}\right] .
$$

LEMMA 1.1. - Let $0<\beta, r<+\infty, \psi_{\mathrm{I}}(t)>0$ for $t \in(0, \beta), \psi_{\mathrm{I}}(t) \in L(0, \beta)$ and assume that $\omega_{1}(t)>0$ is continuous for $t \in[0,+\infty)$,

$$
\int_{0}^{\beta} \psi_{1}(t) d t<\Omega_{1}(0), \quad \int_{0}^{\beta} \Omega_{1}^{-1}\left[\int_{0}^{t} \psi_{1}(\tau) d \tau\right] d t>r,
$$

where

$$
\Omega_{1}(t)=\int_{i}^{+\infty} \frac{d \tau}{\omega_{1}(\tau)}, \quad \Omega_{1}^{-1}\left[\Omega_{1}(t)\right]=t
$$


Then there exists a number $\beta_{1} \in(0, \beta)$, such that

$$
\omega_{1}(t, x)=\psi_{1}(t) \omega_{1}(|x|) \in B_{1}\left(r ; \beta_{1}, \beta\right) .
$$

Proof of lemma 1.1. - According to (1.8) we can find numbers $\varepsilon \in(0$, $+\infty)$ and $\beta_{1} \in(0, \beta)$ such that

$$
\int_{\beta_{1}}^{\beta} \Omega_{1}^{-}\left[\varepsilon+\int^{t} \psi_{1}(\tau) d \tau\right] d t>r .
$$

Let $\beta \in\left(0, \beta_{1}\right)$ and let $u(t)$ be some function, absolutely-continuons together with $u^{\prime}(t)$ on the interval $[\beta, \beta]$ satisfying the inequalities (1.2) and (1.3), where

$$
\omega_{1}(t, y)=\psi_{1}(t) \omega_{1}(|y|) .
$$

We first show that for some $t_{0} \in\left[\beta_{1}, \beta\right]$ we have

$$
\left|u^{\prime}\left(t_{0}\right)\right| \leq \Omega_{1}^{-1}\left[\varepsilon+\int_{0}^{t_{0}} \psi_{1}(\tau) d \tau\right] .
$$

In fact, with the opposite hypothesis we have

$$
\left|u^{\prime}(t)\right| \geq \Omega_{1}^{-1}\left[\varepsilon+\int_{0}^{t} \psi_{1}(\tau) d \tau \mid \text { for } t \in\left[\beta_{1}, \beta\right]\right.
$$

which yields the contradictory result

$$
r \geq\left|u\left(\beta_{1}\right)\right| \geq \int_{\beta_{1}}^{\beta} \Omega_{1}^{-1}\left[\varepsilon+\int_{0}^{t} \psi_{1}(\tau) d \tau\right] d t>r .
$$

According to (1.2) and (1.11) it follows from (1.3) that

$$
\left.\left|u^{\prime}(t)\right|^{\prime} \geq-\psi_{1}(t) \omega_{1} !\left|u^{\prime}(t)\right|\right) \text { for } t \in[\underline{\beta}, \beta] .
$$

Hence, according to (1.9) and (1.12), we can find

$$
\begin{gathered}
\left|u^{\prime}(t)\right| \leq \Omega_{1}^{-1}\left[\Omega_{1}\left(\left|u^{\prime}\left(t_{0}\right)\right|\right)-\int_{i}^{t_{0}} \psi_{1}(\tau) d \tau\right]< \\
<\Omega_{1}^{-1}\left[\varepsilon+\int_{0} \psi_{1}(\tau) d \tau\right] \leq \Omega_{1}^{-1}(\varepsilon) \text { for } t \in\left[\underline{\beta}, t_{0}\right] .
\end{gathered}
$$


Consequently, for $\rho_{1}(t) \equiv Q_{1}^{-1}(\varepsilon)$ the inequality (1.4) holds. Since, in addition, $\varepsilon$ is independent of $\beta$ and $u(t)$, the correctness of Lemma 1.1 is obvious.

Lenma 1.2. - If a function $u(t)$ is continuously differentiable on an interval $[a, b]$ and on this interval satisfies the inequalities

$$
0 \leq u(t) \leq r
$$

then for some $t_{0} \in[a, b]$ we have

$$
\left|u^{\prime}\left(t_{0}\right)\right| \leq \frac{r}{b-a}
$$

Proof of LEMma 1.2. In fact, with the opposite hypothesis we have

$$
\left|u^{\prime}(t)\right|>\frac{r}{b-\alpha} \text { for } t \in[a, b]
$$

which yields the contradictory result

$$
r \geq|u(b)-u(a)|=\int_{a}^{b}\left|u^{\prime}(t)\right| d t>r .
$$

LeMma 1. 3. Let $0<\beta, r<+\infty, \psi_{1}(t)>0$ for $t \in(0, \beta), \psi_{1}(t) \in L(\varepsilon, \beta)$ for every $\varepsilon \in(0, \beta), \omega_{1}(t)>0$ is continuous for $t \in[0,+\infty)$,

$$
\Omega_{1 \delta}(t)=\int_{\delta}^{t} \frac{d \tau}{\omega_{1}(\tau)} \rightarrow+\infty \text { for } t \rightarrow+\infty, \Omega_{1 \delta}^{-1}\left[\int_{t}^{\beta} \psi_{1}(\tau) d \tau\right] \in L(0, \beta)
$$

where

$$
\frac{r}{\beta}<\delta<+\infty, \Omega_{1 \delta}^{-1}\left[\Omega_{1 \delta}(t)\right] \equiv t .
$$

Then we can find such a number $\beta_{1} \in(0, \beta)$ that

$$
\omega_{1}(t, y)=\psi_{1}(t) \omega_{1}(|y|) \in B_{1}\left(r ; \beta_{1}, \beta\right) .
$$

Proof of Lemma 1.3. Let $\beta_{1} \in(0, \beta)$ be so small, that we have

$$
\delta>\frac{r}{\beta-\beta_{1}} \text {. }
$$

Since $\beta \in\left[\beta_{1}, \beta\right]$ according to Lemma 1.2, it follows from (1.2) and (1.16) that

$$
\left|u^{\prime}\left(t_{0}\right)\right|<\delta \text { for some } t_{0} \in\left[\beta_{1}, \beta\right]
$$


I. T. Kiguradse: On the non-negative non-increasing solutions, etc.

According to (1.14), (1.15) and (1.17) it readily follows from (1.13) that

$$
\left|u^{\prime}(t)\right| \leq \Omega_{1 \delta}^{-1}\left[\int_{i}^{t_{0}} \psi_{1}(\tau) d \tau\right] \leq \Omega_{1 \delta}^{-1}\left[\int_{i}^{\beta} \psi_{1}(\tau) d \tau\right] \text { for } t \in\left[\underline{\beta}, \beta_{1}\right]
$$

On the other hand, from (1.14) it is obvious that

$$
\rho_{1}(t)=\Omega_{1 \delta}^{-1}\left[\int_{i}^{\beta} \psi_{1}(\tau) d \tau\right]
$$

satisfies the condition (1.1). Thus Lemma 1.3 is proved.

LEMMA 1.4 Let

$$
\omega_{1}(t, y)=\psi_{1 k}(t) \omega_{1 k}(|y|) \quad \text { for } \quad t \in\left(t_{k-1}, t_{k}\right) \quad(k=1,2, \ldots),
$$

where

$$
0=t_{0}<t_{1}<\ldots, \quad \lim _{k \rightarrow \infty} t_{k}=+\infty, \quad \psi_{1 k}(t) \geq 0, \quad \psi_{1 k}(t) \in L^{P^{k}\left(t_{k-1}, t_{k}\right),}
$$

$\omega_{1 k}(t)>0$ is continuous for $t \in[0,+\infty)$,

$$
1 \leq p_{1 k}<+\infty \quad(k=1,2, \ldots)
$$

$$
\int_{0}^{+\infty} \frac{t^{1 / q_{1 k}}}{\omega_{1 k}(t)} d t=+\infty, \frac{1}{p_{1 k}}+\frac{1}{q_{1 k}}=1 \quad(k=1,2, \ldots) .
$$

Then $\omega_{1}(t, y) \in B_{1}\left(r ; \beta_{1}, \beta\right)$ for every $r \in(0,+\infty), \beta \in(0,+\infty)$ and $\beta_{1} \in(0, \beta)$.

Proof of lemma 1.4. - Let $r, \beta \in(0,+\infty), \beta_{1} \in(0, \beta)$ and $\underline{\beta} \in\left(0, \beta_{1}\right]$ be arbitrary numbers, and $u(t)$-an arbitrary function, absolutely-continuous together with $u^{\prime}(t)$ on the interval $[\beta, \beta]$ and satisfying the inequalities (1.2) and (1.3).

According to (1.2.) and (1.18) it follows from (1.3) that

$$
\left|u^{\prime}(t)\right|^{\prime} \geq-\psi_{1 k}(t) \omega_{1 k}\left(\left|u^{\prime}(t)\right|\right) \text { for } t \in\left(t_{k-1}, t_{k}\right) \cap[\underline{\beta}, \beta] \quad(k=1,2, \ldots) .
$$

According to Lemma 1.2 we have from (1.2)

$$
\left|u^{\prime}\left(t^{*}\right)\right| \leq \frac{r}{\beta-\beta_{1}} \text { for some } t^{*} \in\left[\beta_{1}, \beta\right]
$$

Let

$$
t^{*} \in\left(t_{m-1}, t_{m}\right), \beta \in\left(t_{n-1}, t_{n}\right], 1 \leq m \leq n .
$$


We assume that

$$
\rho_{1}(t)=U_{k}(t) \text { for } t \in\left[t_{k-1}, t_{k}\right] \quad(k=1,2, \ldots, n),
$$

where $\left.U_{k}(t) k=1,2, \ldots, n\right)$ are functions given by the equalities

$$
\begin{gathered}
\int_{r / \beta-\beta_{1}}^{U_{n}(t)} \frac{\tau^{1 / q_{1 n}}}{\omega_{1 n}(\tau)} d \tau=r^{1 / q_{1 n} \eta_{1 n}}(t) \quad \text { for } \quad t \in\left[t_{n-1}, t_{n}\right], \\
\int_{U_{k+1}\left(t_{k}\right)}^{U_{k}(t)} \frac{\tau^{1 / q_{1 k}}}{\omega_{1 k}(\tau)} d \tau=r^{1 / q_{1 k} \eta_{1 k}(t)} \text { for } t \in\left[t_{k-1}, t_{k}\right] \quad(k=n-1, \ldots, 1)
\end{gathered}
$$

and

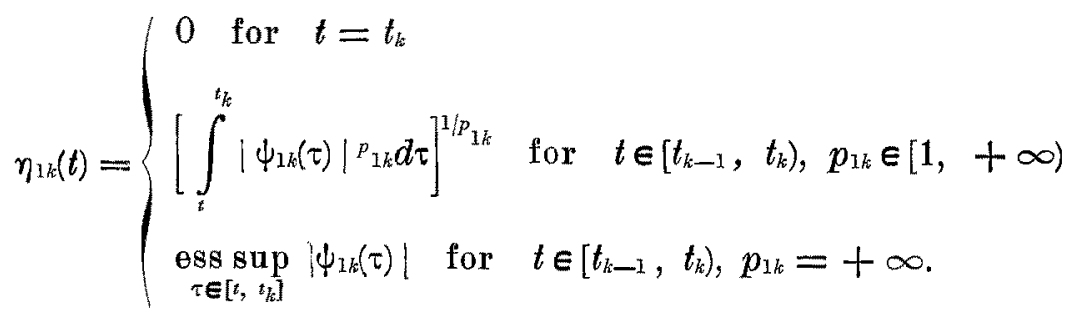

By (1.22) and (1.23) we obtain from (1.21)

$$
\int_{r, \beta-\beta_{1}}^{\left.\mid u^{\prime}(t)\right\}} \frac{\tau^{1 / q_{1 m}}}{\omega_{1 m}(\tau)} d \tau \leq \int_{i}^{t^{*}} \psi_{1 m}(\tau)\left|u^{\prime}(\tau)\right|^{1 / q_{1 m}} d \tau \text { for } t \in\left(t_{m-1}, t^{*}\right] \cap[\beta, \beta] .
$$

Applying the Hölder inequality, according to (1.2) and (1.26) we can find

$$
\begin{gathered}
\int_{i}^{t^{*}} \Psi_{1 m}(\tau)\left|u^{\prime}(\tau)\right|^{1 / q} 1 / x \leq\left\{\int_{i}^{t^{*}}\left|u^{\prime}(\tau)\right| d \tau\right\}^{1 / q_{1 m}} \eta_{1 m}(t) \leq \\
\leq \boldsymbol{r}^{1 / q_{1 m} \eta_{1 m}(t)} \text { for } t \in\left[t_{m-1}, t^{*}\right] \cap[\underline{\beta}, \beta] .
\end{gathered}
$$

Since $U_{m+1}\left(t_{m}\right)>\frac{r}{\beta-\beta_{1}}$, if $m<n$, then, because of (1.25) and (1.28); from (1.27) we obtain

$$
\left|u^{\prime}(t)\right| \leq U_{m}(t) \text { for } t \in\left[t_{m-1}, t^{*}\right] \cap[\beta, \beta]
$$

On the basis of the inequality (1.29), according to (1.21) and (1.25) it is 
easily seen that

$$
\left|u^{o}(t)\right| \leq U_{k}(t) \text { for } t \in\left[t_{k-1}, t_{k}\right] \cap[\underline{\beta}, \beta] \quad(k=m-1, \ldots, 1)
$$

Since $t^{*} \geq \beta$, because of $(1.24)$ it follows from (1.29) and (1.30) that inequality (1.4) holds.

$A s$, in addition, $\rho_{1}(t)$ is continuous on the interval $\left[0, \beta_{1}\right]$ and independent of $\beta$ and $u(t)$, the correctness of Lemma 1.4 is obvious.

LeMma 1.5. - Let

$$
\omega_{2}(t, y)=\psi_{2 k}(t) \omega_{2 k}(|y|) \quad \text { for } t \in\left(\tau_{k-1}, \tau_{k}\right) \quad(k=1,2, \ldots)
$$

where

$$
\begin{aligned}
& \left.0 \leq \tau_{0}<\tau_{1}<\ldots, \lim _{k \rightarrow \infty} \tau_{k}=+\infty, \psi_{2 k}(t) \geq 0, \psi_{2 k}(t) \in L^{p_{2 k}\left(\tau_{k-1}\right.}, \tau_{k}\right), 1 \leq p_{2 k} \leq+\infty \\
& (k=1,2, \ldots) \\
& \omega_{2 k}(t)>0 \text { is continuous for } t \in[0,+\infty), \int_{0}^{+\infty} \frac{t^{1 / q_{2 k}}}{\omega_{2 k}(t)} d t=+\infty \text {, } \\
& \frac{1}{p_{2 k}}+\frac{1}{q_{2 k}}=1 \quad(k=1,2, \ldots) \text {. }
\end{aligned}
$$

Then $\omega_{2}(t, y) \in B_{2}\left(r ; \alpha, \alpha_{1}\right)$ for every $r \in(0,+\infty), \alpha \in\left(\tau_{0},+\infty\right)$ and $\alpha_{1} \in(\alpha,+\infty)$.

Proof of lemma 1.5. - Assuming

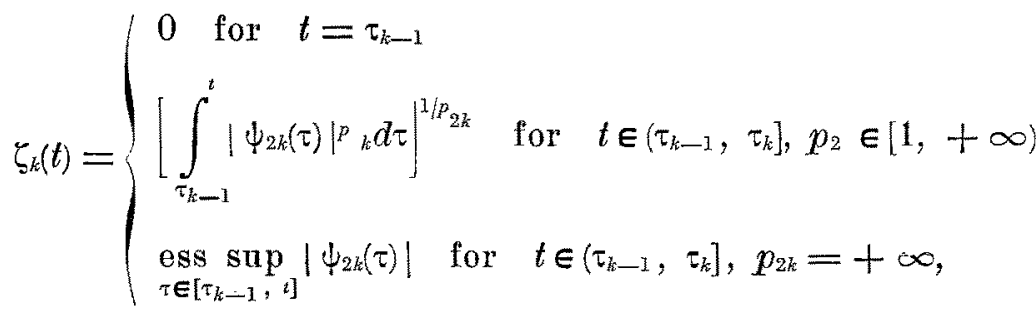

$$
\begin{aligned}
& \int_{r / \alpha_{1}-\alpha}^{N_{1}(t)} \frac{\tau^{1 / q_{21}}}{\omega_{21}(\tau)} d \tau=r^{1 / q_{21}} \zeta_{1}(t) \quad \text { for } \quad t \in\left[\tau_{0}, \tau_{1}\right] \\
& \int_{N_{k-1}\left(\tau_{k-1}\right)}^{N_{k}(t)} \frac{\tau^{1 / q_{2 k}}}{\omega_{2 k}(\tau)}=r^{1 / q_{2 k}} \zeta_{k}(t) \text { for } t \in\left[\tau_{k-1}, \tau_{i k}\right] \quad(k=1,2, \ldots)
\end{aligned}
$$

and

$$
\rho_{2}(t)=N_{k}(t) \quad \text { for } \quad t \in\left[\tau_{k-1}, \tau_{k}\right] \quad(k=1,2, \ldots)
$$


Then, by a reasoning analogous to that of Lemma 1.4, we obtain the following conclusion: for every $\alpha \in\left[\alpha_{1},+\infty\right)$ and for an arbitrary function $u(t)$ which is absolutely-continuous together with $u^{\prime}(t)$ on an interval $\left[\alpha_{1}, \bar{\alpha}\right]$ and satisfies the inequalities (1.5) and (1.6), where $\omega_{2}(t, y)$ is defined by the equality (1.31), estimation (1.7) is true.

Lemma $1.6-$ Let $0<r, a<+\infty$,

$$
\begin{gathered}
\psi_{2}(t) \geq 0, \psi_{2}(t) \in L(a,+\infty), \\
\omega_{2}(t)>0 \text { is continuous for } t \in(0,+\infty) .
\end{gathered}
$$

Then we can find such numbers $\alpha \in(\alpha,+\infty)$, and $\alpha_{1} \in(\alpha,+\infty)$, that $\omega_{2}(t, y)$ $=\psi_{2}(t) \omega_{2}(|y|) \in B_{2}\left(r ; \alpha, \alpha_{1}\right)$.

Proof of LemMa 1.6 - According to (1.34) and (1.35) we can find such numbers $\alpha, \in(a,+\infty)$ and $\rho_{2} \in[r,+\infty)$ that

$$
\int_{r}^{p_{2}} \frac{d t}{\omega_{2}(t)}=\int_{z}^{+\infty} \psi_{2}(t) d t
$$

Put $\alpha_{1}=\alpha+1, \bar{\alpha} \in(\alpha+1,+\infty)$ and $\omega_{2}(t, y)=\psi_{2}(t) \omega_{2}(|y|)$ according to (1.5), froln (1.6) we obtain

$$
\left.\left|u^{\prime}(t)\right|^{\prime} \leq \psi_{2}(t) \omega_{2}\left(\mid u^{\prime}, t\right) \mid\right) \text { for } t \in[\alpha, \bar{\alpha}],
$$

but, on the other hand, by Lemma 1.2 we have

$$
\left|u^{\prime}\left(t^{*}\right)\right| \leq r \text { for some } t_{=}^{*} \in\left[\alpha, \alpha_{1}\right] .
$$

According to (1.35) and (1.38) from (1.37) we can find

$$
\int_{r}^{\left|u^{\prime}(t)\right|} \frac{d \tau}{\omega_{2}(\tau)} \leq \int_{t^{*}}^{t} \psi_{2}(\tau) d \tau \leq \int_{\alpha}^{+\infty} \psi_{2}(\tau) d \tau \text { for } t \in\left[\alpha_{1}, \vec{\alpha}\right] .
$$

Hence, from (1.36), it follows that for $\rho_{2}(t) \equiv \rho_{2}$ inequality (1.7) holds. Lemma 1.6 is thus proved.

\section{§. - Existence theorems}

It is assumed throughout what follows that on a domain $a \leq t \leq b$, $-\rho \leq x, y \leq \rho$ for every $a, b, \rho \in(0,+\infty)$ a function $f(t, x, y)$ satisfies Caratheodory's conditions, i. e., $f(t, x, y)$ is measurable with respect to $t$ on a segment 
$[a, b]$ for every fixed $x, y \in(-\rho, \rho)$, it is continuous with respect to $x, y$ on a domain - $-\leq x, y \leq \rho$ for almost all $t \in[a, b]$ and $f^{*}(t, \rho)=\sup _{x, y \in(-\rho, \rho)}|f(t, x, y)| \in$ $\in L(a, b)$. In addition, generally, $f *(t, \rho) \in L(0, b)$.

A function $u(t)$ is said to be the solution of problem (1)-(2), if it is absolutely-continuous together with $u^{\prime}(t)$ on every segment of the interval $(0,+\infty)$ and on this interval satisfies the equation (1) and the conditions (2).

$$
\begin{aligned}
& \text { Theonem 2.1. - Let } 0<r<+\infty, 0<\alpha<\alpha_{3} \leq \beta_{1}<\beta<+\infty \\
& f(t, 0,0) \equiv 0, \quad f(t, x, 0) \geq 0 \text { for } \quad 0<t, x<+\infty
\end{aligned}
$$

and

$$
f(t, x, y) \geq-\omega_{2}(t, y) \quad \text { for } \quad \alpha<t<+\infty, \quad 0 \leq x \leq r, \quad-\infty<y \leq 0,
$$

where

$$
\omega_{1}(t, y) \in B_{1}\left(r ; \beta_{1}, \beta\right) \text { and } \omega_{2}(t, y) \in B_{2}\left(r ; \alpha, \alpha_{1}\right) .
$$

Then for every $u_{0} \in[0, r]$ problem (1)-(2) possesses at least one solution.

In order to prove Theorem 2.1 the following two Lemmas will be needed.

Lemma 2.1. - Let $0<a<b<+\infty$ and let a function $g(t, x, y)$ for every $p \in(0,+\infty)$ satisfy Caratheodory's conditions on a domain $a \leq t \leq b,-p \leq x$, $y \leq \rho$, when

$$
\begin{gathered}
g(t, x, y) \operatorname{sign} x \geq 0 \text { for } a \leq t \leq b, \quad-\infty<x<+\infty, \quad 0 \leq y<+\infty \\
|g(t, x, y)| \leq g(t) \text { for } \quad a \leq t \leq b, \quad-\infty<x, y<+\infty
\end{gathered}
$$

where

$$
g(l) \in L(a, b)
$$

Then, for every $u_{0} \in[0,+\infty)$ the differential equation

$$
\left.u^{\prime \prime}=g^{\prime} t, u, u^{\prime}\right)
$$

has a solution $u(t)$ which satisfies the boundary conditions

$$
u(a)=u_{0}, \quad u(b)=0
$$

and the inequalities

$$
u(t) \geq 0 \quad u^{\prime}(t) \leq 0 \quad \text { for } \quad a \leq t \leq b .
$$


Proof of Lemira 2.1 - Let $C[a, b]$ be the space of continuously differentiable functions on a segment $[a, b]$ with the norm

$$
\|u(t)\|=\max _{i \in[a, b]}\left(|u(t)|+\left|u^{\prime}(t)\right|\right)
$$

and let $S$ be a set of this space, defined in the following way:

$$
u(t) \in S \quad \text { if } \quad\|u(t)\| \leq 2 u_{0}+(b-a+1) \int_{a}^{b} g(t) d t .
$$

We now eonsider the operator

$$
\begin{gathered}
\left.H u(t)=\frac{b-t}{b-a} u_{0}+\frac{t-b}{b-a} \int_{a}^{t}(\tau-a) g^{\prime} \tau, u(\tau), u^{\prime}(\tau)\right) d \tau+ \\
+\frac{t-a}{b-a} \int_{t}^{b}\left(\tau-b g\left(\tau, u(\tau), u^{\prime}(\tau)\right) d \tau\right.
\end{gathered}
$$

Because of (2.5), (2.10) and (2.11) it follows from (2.12) that, if $u(t) \in S$ then

$$
v(t)=H u(t) \in S \quad \text { and } \quad\left|v^{\prime}\left(t_{2}\right)-v^{\prime}\left(t_{1}\right)\right| \leq\left|\int_{t_{1}}^{t_{2}} g(\tau) d \tau\right| \text { for } t_{1}, t_{2} \in[a, b]
$$

According to the Arzela-Ascoli lemma it follows from (2.6), (2.11) and (2.13) that $H S$ is a compact set of the space $O[a, b]$.

Therefore, the operator $H$ transforms the set $S$ into a compact subset. On the other hand, it is easily seen that $H$ is a continuous operator. Consequently, according to the Schauder Theorem (cf., [3], pp. 574-578), we can find a function $u(t) \in S$ such that

$$
u(t)=H u(t) \quad \text { for } \quad t \in[a, b]
$$

From $(2 \cdot 12)$ and $(2.14)$ it is clear that $u(t)$ is the solution of problem (2.7) $-(2-8)$.

We have to prove now that the inequalities (2.9) are satisfied. We first show that

$$
u(t) \geq 0 \text { for } t \in[a, b]
$$

Under the opposite assumption, since $u(a)=u_{0} \geq 0$ and $u(b)=0$, we can 
find such numbers $t_{1}, t_{2} \in(a, b)$, that

$$
u(t)<0, u^{\prime}(t)>0 \text { for } t_{1}<t \leq t_{2}
$$

and

$$
u^{\prime}\left(t_{1}\right)=0 .
$$

On the other hand, by (2.4) and (2.16) it follows from (2.7) that

$$
u^{\prime \prime}(t) \leq 0 \quad \text { for } \quad t_{1} \leq t \leq t_{2}
$$

and therefore $u^{\prime}\left(t_{1}\right) \geq u^{\prime}\left(t_{2}\right)>0$, but this contradicts the condition (2.17).

Thus, in order to prove this lemma it only remains to be shown that

$$
u^{\prime}(t) \leq 0 \text { for } t \in[a, b]
$$

Under the opposite assumption, by (2.8) and (2.15) we can find such numbers $t_{1}, t_{2} \in(a, b)$ that

$$
u^{\prime}(t)>0 \text { for } t_{1} \leq t<t_{2} \text { and } u^{\prime}\left(t_{2}\right)=0,
$$

which is again a contradiction, but by (2.4) and (2.15) it follows from (2.7) $u^{\prime \prime}(t) \geq 0$ for $t_{1} \leq t \leq t_{2}$.

The contradiction thus obtained proves the Lemma.

LEMma 2.2. - If $\left\{v_{k}(t)\right\}$ is a sequence of functions which are uniformly bounded and equicontinuous on each segnent of the interval $(0,+\infty)$, then this sequence, contains a subsequence, which is uniformly convergent on each segment contained within $(0,+\infty)$.

Proof of uemra 2.2 - According to the Arzela-Ascoli lemma, the sequence $\left\{v_{k}(t)\right\}$ contains a subsequence $\left\{v_{1 k}(t)\right\}$ which is uniformly convergent on the segment $\left[\frac{1}{2}, 1\right]$.

In its turn $\left\{v_{1 k}(t)\right\}$ contains a subsequence $\left\{v_{2 k}(t)\right\}$ which is uniformly convergent on the segment $\left[\frac{1}{3}, 2 \mid\right.$ etc., proceeding further same way we obtain the system of sequences $\left\{v_{i k}(t)\right\}(i=1,2, \ldots)$ such that

$$
\left\{v_{1 k}(t)\right\} \supset\left\{v_{2 k}(t)\right\} \supset \ldots
$$

and each sequence $\left\{v_{i k}(t)\right\}$ is uniformly convergent on the segment $\left[\frac{1}{1+i}, i\right]$. Proceeding from this, it can readily be verified that $\left\{v_{k k}(t)\right\}$ is uniformly convergent on each segment of $(0,+\infty)$. 
Proof of THEOREM 2.1 - Let the functions $\rho_{1}(t)$ and $\rho_{2}(t)$ be chosen according to the definitions 1.1 and 1.2.

We introduce the following notation

$$
\begin{gathered}
U\left(t_{*}, t^{*}\right)=r+\max _{t \in\left[t_{*}, \beta\right]} \rho_{1}(t)+\max _{t \in\left[R, t^{*}\right]} \rho_{2}(t), \\
\xi(t ; \tau)=\left\{\begin{array}{l}
1 \text { for } 0 \leq t \leq \tau \\
2-\frac{t}{\tau} \text { for } \tau<t<2 \tau \\
0 \text { for } t \geq 2 \tau,
\end{array}\right. \\
\sigma_{k}(t)=\left\{\begin{array}{ll}
t & \text { for }(-1)^{k-1} t>0 \\
0 & \text { for }(-1)^{k-1} t \leq 0
\end{array} \quad(k=1,2) .\right.
\end{gathered}
$$

We put $a=\frac{\alpha}{k}, b=\beta+k$ and

$$
g(t, x, y)=\xi\left[|x|+|y| ; U\left(\frac{1}{k}, \beta+k\right)\right] f\left(t, \sigma_{1}(x), \sigma_{2}(y)\right),
$$

by (2.1), (2.19) and (2.20) we can easily verify that all the conditions of Lemma 2.1 are satisfied. Therefore the equation (2.7) has the solution $u_{k}(t)$ satisfying the boundary conditions

$$
u_{i}\left(\frac{\alpha}{k}\right)=u_{0}, u_{k}(\beta+k)=0
$$

and the inequalities

$$
u_{k}(t) \geq 0, \quad u_{k}^{\prime}(t) \leq 0 \quad \text { for } \quad t \in\left[\frac{\alpha}{k}, \beta+k\right] .
$$

By (2.2), (2.19), (2.20), (2.21) and (2.23) it follows from (2.7) that

$$
\begin{gathered}
u_{k}^{\prime \prime}(t) \leq \omega_{1}\left(t, u_{k}^{\prime}(t)\right) \text { for } t \in\left[\frac{\alpha}{k}, \beta\right], \\
u_{k}^{\prime \prime}(t) \geq-\omega_{2}\left(t, u_{k}^{\prime}(t)\right) \text { for } t \in[\alpha, \beta+k] .
\end{gathered}
$$

Hence, by the conditions (2.3) and definitions 1.1 and 1.2 it follows that

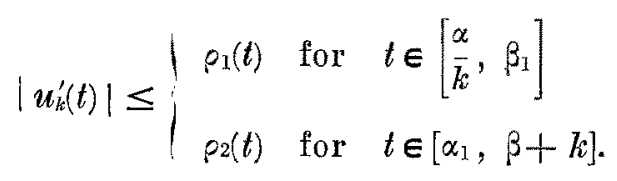

Since $u_{0} \in[0, r]$, by $(2.23)$ we have

$$
0 \leq u_{k}(t) \leq r \text { for } \quad t \in\left[\frac{\alpha}{k}, \beta+k\right]
$$


Inasmuch as $\alpha_{1} \leq \beta_{1}$, from (2.18), (2.24) and $(2.25)$ we have

$$
\left|u_{k}(t)\right|+\left|u_{k}^{\prime}(t)\right| \leq U\left(\frac{\alpha}{k}, \beta+k\right) \text { for } t \in\left[\frac{\alpha}{k}, \beta+k\right] .
$$

By $\left(2.19,(2.20),(2.21),(2.23)\right.$ and $(2.26)$ it follows from $(2.7)$ that $u_{k}(t)$ is a solution of the equation (1).

Thus, the equation (1) has a sequence of solution $\left\{u_{k}(t)\right\}$, defined, corre. spondingly, on the intervals $\left[\frac{\alpha}{\bar{k}}, \beta+k\right](k=1,2, \ldots)$ satisfying the conditions (2.22), (2.23), (2.24) and (2.25).

Putting

$$
v_{k}(t)=\left\{\begin{array}{l}
u\left(\frac{\alpha}{k}\right)+\left(t-\frac{\alpha}{k}\right) u^{\prime}\left(\frac{\alpha}{k}\right) \text { for } t \in\left[0, \frac{\alpha}{k}\right) \\
u(t) \text { for } t \in\left[\frac{\alpha}{k}, \beta+k\right] \\
u(\beta+k)+(t-\beta-k) u^{\prime}(\beta+k) \text { for } t \in(\beta+k,+\infty)
\end{array}\right.
$$

we can easily conclude that for every $t_{*} \in(0, \alpha]$ and $t^{*} \in[\beta,+\infty)$ we have

$$
\left|v_{k}(t)\right|+\left|v_{k}^{\prime}(t)\right| \leq\left(1+t^{*}\right) U\left(t_{*}, t^{*}\right) \text { for } t \in\left[t_{*}, t^{*}\right]
$$

and

$$
\begin{gathered}
\left|v_{k}\left(t_{2}\right)-v_{k}\left(t_{1}\right)\right|+\left|v_{k}^{\prime}\left(t_{2}\right)-v_{2}^{\prime}\left(t_{1}\right)\right| \leq \\
\leq U\left(t_{*}, t^{*}\right)\left|t_{2}-t_{1}\right|+\left|\int_{t_{1}}^{t_{2}} f^{*}(t) d t\right| \text { for } t_{1}, t_{2} \in\left[t_{*}, t^{*}\right]
\end{gathered}
$$

where

$$
f^{*}(t)=\max _{|x|+|y| \leq\left(1+t^{*}\right) U\left(t_{*}, t^{*}\right)}|f(t, x, y)|
$$

Consequently, $\left\{v_{k}(t)\right\}$ and $\left\{v_{k}^{\prime}(t)\right\}$ are uniformly bounded and equicontinuos on the interval $\left[t_{*}, t^{*}\right]$.

By Lemma 2.2 it is clear that $\left\{v_{k}(t)\right\}$ contains a subsequence $\left\{v_{k_{i}}(t)\right\}$, such that $\left\{v_{k_{i}}(t)\right\}$ and $\left\{v_{k_{i}}^{\prime}(t)\right\}$ are uniformly convergent on each segment of $(0,+\infty)$. We put

$$
\lim _{i \rightarrow \infty} v_{k_{i}}(t)=u(t) .
$$

Clearly,

$$
\lim _{i \rightarrow \infty} v_{k_{i}}^{\prime}(t)=u^{\prime}(t)
$$


By (1.1), (2.22), (2.23), (2.24) and (2.27) we have

$$
\begin{aligned}
& \left|v_{k_{i}}(t)-u_{0}\right| \leq \int_{0}^{t} \rho_{1}(\tau) d \tau \text { for } t \in\left[\frac{\alpha}{\bar{k}_{i}}, \beta_{1}\right], \\
& v_{k_{i}}(t) \geq 0, \quad v_{k_{i}}^{\prime}(t) \leq 0 \quad \text { for } \quad t \in\left[\frac{\alpha}{k_{i}}, \beta+k_{i}\right]
\end{aligned}
$$

and

$$
v_{k_{i}}^{\prime}(t)=v_{k_{i}}^{\prime}(\alpha)+\int_{\alpha}^{t} f\left(\tau, v_{k_{i}}(\tau), v_{k_{i}}^{\prime}(\tau)\right) d \tau \text { for } t \in\left[\frac{\alpha}{k_{i}}, \beta+k_{i}\right]
$$

By (2.28) and (2.29) it follows from (2.30) that $u(t)$ satisfies the conditions (2), and for $i \rightarrow \infty(2.31)$ becomes

$$
u^{\prime}(t)=u^{\prime}(\alpha)+\int_{\alpha}^{t} f\left(\tau, u(\tau), u^{\prime}(\tau)\right) d \tau \text { for } t \in(0,+\infty)
$$

Consequently, $u(t)$ is the solution of problem (1)-(2). This proves Theorem 2.1.

By the Lemmas 1.1, 1.3 and 1.5 from Theorem 2.1 there directly follows

Theorem 2.2 - Let the conditions (2.1) be satisfied, when $0<r, \beta<+\infty$,

$$
f(t, x, y) \leq \bigcup_{1}(t) \omega_{1}(|y|) \quad \text { for } \quad 0<t<\beta, \quad 0 \leq x \leq r, \quad-\infty<y \leq 0,
$$

$\psi_{2}(t)>0, \psi_{1}(t) \in L(\varepsilon, \beta)$ for every $\varepsilon \in(0, \beta)$ and either the condilions (1.8) or the conditions (1.14) are satisfied, where $\Omega_{1}^{-1}(t), \Omega_{1 \delta}^{-1}(t)$ and $\delta$, correspondingly, satisfies the conditions (1.9) and (1.15). Let, in addition,

$$
\begin{aligned}
& f(t, x, y) \geq-\psi_{2 k^{\prime}}(t) \omega_{2 k}(|y|) \text { for } t_{k-1}<t<t_{k}, \\
& 0 \leq x \leq r, \quad-\infty<y \leq 0, \\
& 0=t_{0}<l_{1}<\ldots, \quad \lim _{k \rightarrow \infty} t_{k}=+\infty, \quad \biguplus_{2 k}(t) \geq 0, \\
& 1 \leq p \leq+\infty(k=1,2, \ldots)
\end{aligned}
$$

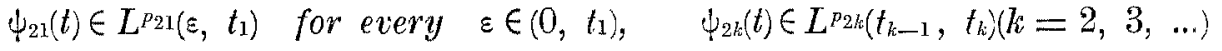

and let the functions $\omega_{2 k}(t)(k=1,2, \ldots)$ satisfy the conditions (1.33). Then for every $u_{0} \in\left[0, v_{1}\right.$ problem $(1) \cdot(2)$ possesses at least one solution.

It can easily be verified that Theorem 2.2 has 
Corollarx 1 - Let the conditions (2.1) be satisfied, $0<r, \beta<+\infty$ and $f(t, x, y) \leq \psi_{1}(b)\left(1+|y|^{\lambda}\right.$ for $0<t<\beta, \quad 0 \leq x \leq r, \quad-\infty<y \leq 0$, where $\psi_{1}(t)$ and $\lambda$ satisfy one of the following three conditions $\left(^{1}\right)$ $\in L(0, \beta)$.

1) $1<\lambda<+\infty, \psi_{1}(t)>0, \psi_{1}(t) \in L(0, \beta)$ and $\int_{0}^{\beta}\left[\int_{0}^{t} \psi_{1}(\tau) d \tau\right]^{1 / 1-\hat{\lambda}} d t=+\infty$ $\in L(0, \beta)$;

2) $\lambda=1, \psi_{1}(t)>0, \psi_{1}(t) \in L(\varepsilon, \beta)$ for every $\varepsilon \in(0, \beta)$ and $\exp \left[\int_{i}^{\beta} \psi_{1}(\tau) d \tau\right] \epsilon$

3) $-\infty<\lambda<1, \psi_{1}(t)>0, \psi_{1}(t) \in L(\varepsilon, \beta)$ for every $\varepsilon \epsilon(0, \beta)$ and $\left[\int_{i}^{\beta} \psi(\tau) d \tau\right]^{1 / 1-\lambda} \epsilon$ If, in addition,

$$
\begin{gathered}
f(t, x, y) \geq-\psi_{2 k}(t)(1+|y|)^{1+1 / q_{2 k}} \text { for } t_{k-1}<t<t_{k}, \\
0 \leq x \leq r, \quad-\infty<y \leq 0, \quad \frac{1}{p_{2 k}}+\frac{1}{q_{2 k}}=1(k=1,2, \ldots)
\end{gathered}
$$

and the conditions (2.33) hold, then for every $u_{0} \in[0, r]$ problem (1)-12) possesses at least one solution.

The above restriations on the function $f(t, x, y)$ with respect to $t$ and $y$, are essential for the solvability of problem (1)-(2). A confirmation of this fact is seen in the following corollary of Theorem 2.2:

Corolliary 2, - Let $f(t, 0,0) \equiv 0$ and

$$
\begin{aligned}
& \psi(t)\left[c_{1}(x)+|y|^{\lambda}\right] \leq f(t, x, y) \leq \psi(t) c_{2}(x)(1+|y|)^{\lambda} \\
& \text { for } 0<t, x<+\infty, \quad-\infty<y \leq 0
\end{aligned}
$$

where $1<\lambda<+\infty, c_{1}(x)$ and $c_{2}(x)$ are continuous and positive on the interval $[0,+\infty)$

$$
\left.\psi(t)>0, \quad \psi(t) \in L(0, \beta) \text { and } \mid \int_{0}^{t} \psi(\tau) d \tau\right]^{1 / \lambda-\lambda} \in L(\beta,+\infty) \text { for } \beta \in(0,+\infty) .
$$

Then for the solvability of problem (1)-(2) it is necessary and sufficient that

$$
\int_{0}^{\beta}\left[\int_{0}^{t} \psi(\tau) d \tau\right]^{1 / 1-\lambda} d t=+\infty \text { for } \beta \in(0,+\infty)
$$

(1) Let $\psi_{1}(t)$ designate, for example, the function $A t^{\lambda-2}|\ln t| \lambda-1-\varepsilon$, where $A=$ const $>0$, $\equiv>0$ for $\lambda \leq 1$ and $\varepsilon=0$ for $\lambda>1$. 
Proof of oORollary 2. - Sufficiency immediately follows from Corollary 1. We have now to prove the necessity. We assume the contrary, i. e., that the condition (2.36) does not hold and problem $(1) \cdot(2)$ is solvable for every $u_{0} \in[0,+\infty)$.

Since under this assumption (2.36) no longer holds, it follows from (2.35) that

$$
U=\int_{0}^{+\infty}\left[\int_{0}^{t} \psi(\tau, d \tau]^{1 / 1-\lambda} d t<+\infty\right.
$$

Hence it is clear that

$$
\int_{0}^{+\infty} \psi(t) d t=+\infty
$$

We now assume that

$$
u_{0}>(\lambda-1)^{1 / 1-\lambda} U
$$

and that $u(t)$ is solution of problem (1)-(2). We have now to prove that

$$
\lim _{t \rightarrow+\infty} u(t)=0
$$

Indeed, under the contrary assumption

$$
\lim _{h \rightarrow+\infty} u(t)=u_{1}>0
$$

we obtain, by (2) and (234), from (1) that

$$
\left|u^{\prime}(1)\right|=\int_{1}^{+\infty}\left|u^{\prime \prime}(\tau)\right| d \tau \geq c_{0} \int_{1}^{+\infty} \psi(\tau) d \tau, \quad c_{0}=\min _{u_{1} \leq x \leq u_{0}} c_{1}(x),
$$

bat this contradicts the condition (2.38).

Because of (2) and (2.34) it follows from (1)

$$
\left|u^{\prime}(t)\right|^{\prime} \leq-\psi(t)\left|u^{\prime}(t)\right|^{2} \text { for } \quad t \in(0,+\infty),
$$

whence it follows directly that

$$
\mid u^{\prime}(t) ! \leq(\lambda-1)^{\frac{1}{1-\lambda}}\left[\int_{0}^{t} \psi(\tau) d \tau\right]^{\frac{1}{1-\lambda}} \text { for } \quad t \in(0,+\infty)
$$


By (2), 2.39) and (2.40) it follows from the last inequality that

$$
u_{0}=\int_{0}^{+\infty}\left|u^{\prime}(t)\right| d t<(\lambda-1)^{\frac{1}{\lambda-1}} U
$$

but this contradicts the inequality (2.39).

This contradiction proves that if $u_{0}$ satisfies the inequality (2.39), then problem (1) - (2) has no solution.

Corolmary 3. - Let $f(t, 0,0) \equiv 0,0<r, \beta<+\infty$ and

$$
\begin{aligned}
& \psi(t, x)(1+|y|)^{\lambda} \leq f(t, x, y) \leq c \psi(t, x)(1+|y|)^{\lambda} \\
& \text { for } 0<t<\beta, \quad 0 \leq x \leq r, \quad-\infty<y \leq 0,
\end{aligned}
$$

where $-\infty<\lambda<1,1 \leq c<+\infty$ and a function $\psi(t, x)$ is non-negative and non-decreasing wilh respect to $x$ and $\psi(t, x) \in L(\varepsilon, \beta)$ for every $\varepsilon \in(0, \beta)$ and $x \in[0, r)$. Then, for the solvability of problem (1).(2) for every $u_{0} \in[0, r)$ it is necessary and sufficient that for every $x \in[0, r)$

$$
\left[\int_{t}^{\beta} \psi(\tau, x) d \tau\right]^{\frac{1}{1-\lambda}} \in L(0, \beta)
$$

Proof of oorollary 3. - Sufficiency follows directly from corollary 1. In order to prove the necessity it is sufficient to show that if for some $x_{0} \in(0, r)$

$$
\int_{0}^{\beta}\left[\int_{i}^{\beta} \psi\left(\tau, x_{0}\right) d \tau\right]^{\frac{1}{1-\lambda}} d t=+\infty
$$

then for $x_{0}<u_{0}<r$ problem (1)-(2) has no solution.

We assume the contrary, i. e. that problem (1).(2) has the solution $u(t)$.

According to (2) and (2.41) we can find such a number $\delta \in(0, \beta)$ that

$$
\left|u^{\prime}(t)\right|^{\prime} \leq-\psi\left(t, x_{0}\right)\left(1+\left|u^{\prime}(t)\right|\right)^{\lambda} \text { for } t \in(0, \delta]
$$

Hence it is clear that

$$
\left|u^{\prime}(l)\right| \geq\left[(1-\lambda) \int_{i}^{\delta} \psi\left(\tau, x_{0}\right) d \tau\right]^{\frac{1}{1-\lambda}} \text { for } \iota \in(0, \delta] .
$$

By (2) and (2.41) it follows from the last inequality that

$$
u_{0}=u(\delta)-u^{\prime}(\delta) \delta+\int_{0}^{\delta}\left|u^{\prime}(t)\right| d t>(1-\lambda)^{\frac{1}{1-\lambda}} \int_{0}^{\delta}\left[\left.\int_{i}^{\delta} \psi\left(\tau, x_{0}\right) d \tau\right|^{\frac{1}{1-\lambda}} d t=+\infty .\right.
$$


This contradiction confirms Corollary 3.

By Lemmas 1.4, 1.5 and 1.6 from Theorem 2.1 we obtain

Thenem 2.3 - Let the condilions (2.1) be fulfilled, i.e. $0<r<+\infty$,

$$
\begin{aligned}
& f(t, x, y) \leq \psi_{1 k}(t) \omega_{1 k}(|y|), \text { for } \quad t \in\left(t_{k-1}, t_{k}\right) \\
& 0 \leq x \leq r, \quad-\infty<y \leq 0 \quad(k=1,2, \ldots)
\end{aligned}
$$

where $t_{k}, \psi_{1 k}(t)$ and $\omega_{1 k}(t)(k=1,2, \ldots)$ satisfy the conditions (1.19) and (1.20). If apart from this, either

$$
\begin{aligned}
& f(t, x, y) \geq-\psi_{2 k}(t) \omega_{2 k}(|y|) \quad \text { for } \quad t \in\left(\tau_{k-1}, \tau_{k}\right), \\
& 0 \leq x \leq 1, \quad-\infty<y \leq 0, \quad(k=1,2, \ldots)
\end{aligned}
$$

where $\tau_{k}, \psi_{2 k}(t)$ and $\omega_{2 k}(t)$ satisfy the conditions (1.32) and (1.33), or

$$
\begin{gathered}
f(t, x, y) \geq-\psi_{2}(l) \omega_{2}(|y|) \text { for } a \leq t<+\infty \\
0 \leq x \leq r, \quad-\infty<y \leq 0,
\end{gathered}
$$

where $a \in[0,+\infty) ; \psi_{2}(t)$ and $\omega_{2}(t)$ satisfy the conditions $(1.34)$ and $(1.35)$, then for every $u_{0} \in[0, r]$ problem (1)-(2) has at least one solution.

THEOREM 2.4. Let $0<r, a<+\infty$,

$$
\begin{gathered}
f, t, x, y) \leq \psi_{1}(t) \quad \text { for } \quad 0<t<a, \quad 0 \leq x \leq r, \quad-r \leq y \leq 0, \\
\psi_{1}(t) \in L(0, a)
\end{gathered}
$$

and let the conditions (2.1), (2.32), (2.33) and (1.33) be satisfied. Then we can find such a number $r_{0} \in(0, r]$ that for every $u_{0} \in\left[0, r_{0}\right]$ problem (1).(2) has at least one solution.

Proof OF THEOREM 2.4. - According to (2.32), (2.33) and (1.33) it is clear that

$$
f(\mathrm{t}, x, y) \geq-\psi_{2}(t) \quad \text { for } \quad 0 \leq t \leq a, \quad 0 \leq x \leq r, \quad-r \leq y \leq 0,
$$

where

$$
\psi_{2}(t)^{\circ} \in L(\varepsilon, a) \text { for every } \varepsilon \in(0, a) \text {. }
$$

By (2.44) and (2.46) we can find such numbers $\beta \in(0, a), \beta_{1} \in(0, \beta)$ and 
$r_{0} \in(0, r]$ that

$$
\frac{r_{0}}{\beta-\beta_{1}}+\int_{0}^{\beta} \psi_{1}(\tau) d \tau<\frac{r}{2} \text { and } \frac{r_{0}}{\beta-\beta}+\int^{\beta} \psi_{2}(\tau) d \tau<\frac{r}{2} .
$$

Consider the equation (2.7), where

$$
g(t, x, y)=\left\{\begin{array}{l}
\xi\left(|y| ; \frac{r}{2}\right) f(t, x, y) \text { for } t \in(0, \beta) \\
f(t, x, y) \text { for } t \in[\beta,+\infty)
\end{array}\right.
$$

and $\xi(t ; \tau)$ is a function defined by the equality (2.19).

By the conditions of theorem 2.4 it lollows easily from (2.19) and (2.48) that for the equation (2.7) all the conditions of Theorem 2.2 are fulfilled and, consequently, problem (2.7)-(2) is solvable for every $u_{0} \in[0, r]$.

Suppose now that $u_{0} \in\left[0, r_{0}\right]$ and let $u(t)$ be the solution of problem $(2.7) \cdot(2)$. According to Lemma 1.2 we have

$$
\left|u^{\prime}\left(t^{*}\right)\right| \leq \frac{r_{0}}{\beta-\beta_{1}} \text { for some } t^{*} \in\left[\beta_{1}, \beta\right]
$$

On the other hand, on the basis of (2), (2.43), (2.45), (2.48) and (2.19) from (2.7) we can find

$$
-\psi_{1}(t) \leq\left|u^{\prime}(t)\right|^{\prime} \leq \psi_{2}(t) \text { for } t \in(0, \beta) \text {. }
$$

Hence, by (2.47) and (2.49) it readily follows that

$$
\left|u^{\prime}(t)\right| \leq \frac{r}{2} \text { for } t \in[0, \beta] .
$$

According to the last inequality from (2.19) and (2.48) it is clear that $u(t)$ is the solution of the equation (1). Theorem 2.4 is thus proved.

\section{\$. - On the uniqueness of the solution of the problem (1)-(2)}

We first state the following theorem on the behaviour of solutions of problem (1)-(2) for $t \rightarrow+\infty$

ThEOREM 3.1. - Let

$$
f(t, x, y) \geq 0 \text { for } \quad 0 \leq t<+\infty, \quad 0 \leq x \leq r, \quad-\infty<y \leq 0
$$

and

$$
f(t, x, y) \geq \sigma(t, x) \text { for } \quad 0<t<+\infty, \quad 0 \leq x \leq r, \quad-r \leq t y \leq 0 \text {, }
$$


where $\sigma(t, x)$ is non-decreasing with respect to $x$,

$$
\sigma(t, x) \in L(a, b) \text { for every } a, b \in(0,+\infty)
$$

and

$$
\int_{0}^{+\infty} t \sigma(t, x) d t=+\infty \text { for every } x \in(0, r]
$$

Then for every $u_{0} \in[0, r]$ and for an arbitrary solution $u(t)$ of problem (1)-(2) we have

$$
\lim _{t \rightarrow+\infty} u(l)=0, \quad \lim _{t \rightarrow+\infty} t u^{\prime}(t)=0
$$

Proof of theorem 3.1. By $(3.1)$ it is clear that if $u_{0} \in[0, r]$, then for an arbitrary solution $u(t)$ of problem $(1)-(2$, we have

$$
0 \leq u(t) \leq r \text { for } t \in(0,+\infty)
$$

and

$$
u^{\prime}(t) \leq 0, \quad u^{\prime \prime}(t) \geq 0 \text { for } \quad t \in(0,+\infty)
$$

Therefore, it follows from the equality

$$
u(0)=u(t)-\int_{0}^{t} u^{\prime}(\tau) d \tau
$$

that

$$
-r \leq t u^{\prime}(t) \leq 0 \text { for } t \in(0,+\infty)
$$

According to $(3.2,(3.5)$ and $(3.7)$ it is clear that

$$
u^{\prime \prime}(t) \geq \sigma(t, u(t)) \text { for } t \in(0,+\infty)
$$

We now assume that

$$
u(t) \geq x_{0} \text { for } t \in(0,+\infty)
$$

Then, since $\sigma(t, x)$ is non-decreasing with respect to $x$ we can find from (3.8) that

$$
u^{\prime \prime}(t) \geq \sigma\left(t, x_{0}\right) \text { for } t \in(0,+\infty)
$$


By (3.5), (3.6) and (3.9) it follows from the equality

$$
u_{0}=u(t)-t u^{\prime}(t)+\int_{0}^{t} \tau u^{\prime \prime}(\tau) d \tau
$$

that

$$
r \geq \int_{0}^{t} \tau \sigma\left(\tau, x_{0}\right) d \tau \text { for } t \in(0,+\infty)
$$

which contradicts condition (3.3). This contradiction proves that

$$
\lim _{t \rightarrow+\infty} u(t)=0
$$

By (3.5) and (3.6) it is elear from (3.10) that there exists a finite limit $\lim t u^{\prime}(t)$. On the other hand, since $u(t)$ is a bounded function, clearly, this $\lim _{t \rightarrow+\infty}$

limit equals zero. Theorem 3.1 is thus proved.

Theorem 3.2. - Let the conditions of theorem 3.1 be fulfilled and

$$
\begin{gathered}
f\left(t, x_{2}, y_{2}\right)-f\left(t, x_{1}, y_{1}\right) \geq l_{1}(t)\left(x_{2}-x_{1}\right)+l_{2}(t)\left(y_{2}-y_{1}\right) \\
\text { for } \quad 0<t<+\infty, \quad 0 \leq x_{1}<x_{2} \leq r, \quad-\frac{r}{t} \leq y_{1} \leq y_{2} \leq 0,
\end{gathered}
$$

where $l_{k}(t) \in L(a, b)(k=1,2)$ for every $a, b \in(0,+\infty)$; let for every $t_{0} \in(0,+\infty)$ and for an arbitrary solution $v(t)$ of the equation

$$
v^{\prime \prime}=l_{1}(t) v+l_{2}(t) v^{\prime}
$$

satisfying the conditions

$$
v\left(t_{0}\right) \geq 0, \quad v^{\prime}\left(t_{0}\right)>0
$$

we have

$$
v(t)>0, \quad v^{\prime}(t)>0 \text { for } t \in\left(t_{0},+\infty\right)
$$

Then for every $u_{0} \in[0, r]$ problem (1)-(2) has only one solution.

Proof of THWorem 3.2. - We assume the contrary, i.e., that for some $u_{0} \in[0, r]$ problem $(1) \cdot(2)$ has two different solution $u_{1}(t)$ and $u_{2}(t)$. It is 
easily seen that

$$
0 \leq u_{i}(t) \leq r, \quad-\frac{r}{t} \leq u_{i}^{\prime}(t) \leq 0 \quad \text { for } \quad t \in(0,+\infty) \quad(i=1,2) \cdot
$$

We put

$$
w(t)=u_{2}(t)-u_{1}(t)
$$

Without loss of generality we can assume that

$$
\sup _{i \in(0,+\infty)}|w(t)|>0
$$

On the other hand, according to Theorem 3.1, it is clear that

$$
\lim _{t \rightarrow 0} w(t)=\lim _{t \rightarrow+\infty} w(t)=0 .
$$

By (3.17) and (3.18), we can obviously find such numbers $t_{0} \in(0,+\infty)$ and $t_{1} \in\left(t_{0},+\infty\right)$ that

$$
w(t)>0, w^{\prime}(t)>0 \text { for } t_{0} \leq t<t_{1}
$$

and

$$
w^{\prime}\left(t_{1}\right)=0 .
$$

By (3.11), (3.15), (3.16) and (3.19) we have

$$
w^{\prime \prime}(t)=l_{1}(t) w(t)+l_{2}(t) w^{\prime}(t)+h(t)
$$

where

$$
\begin{gathered}
h(t)=f\left(t, u_{2}(t), u_{2}^{\prime}(t)\right)-f\left(t, u_{1}(t), u_{1}^{\prime}(t)\right)-l_{1}(t) w(t)-l_{2}(t) w^{\prime}(t) \geq 0 \\
\text { for } t_{0} \leq t \leq t_{1} .
\end{gathered}
$$

From (3.21) it is clear that

$$
w^{\prime}\left(t,=v^{\prime}(t)+\int_{t_{0}}^{t} \frac{\hat{c} \mathcal{J}(t, \tau)}{\partial t} h(\tau) d \tau,\right.
$$

where $\tilde{d r}(t, \tau)$ is the Cauchy function of the equation (3.12) and $v(t)$ is a solution of this equation, satisfying the initial conditions

$$
v\left(t_{0}\right)=w\left(t_{0}\right), \quad v^{\prime}\left(t_{0}\right)=w^{\prime}\left(t_{0}\right) .
$$


It is clear from (3.20) and (3.24) that the inequalities (3.13) are fulfilled. Therefore, according to the assumption of this Theorem, the inequalities (3.14) hold.

For the same reason we have

$$
\frac{\partial \mathcal{d}(t, \tau)}{\partial t} \geq 0 \text { for } t \geq \tau \text {. }
$$

By (3.14), (3.22) and (3.25) from $(3,23)$ we can find

$$
w^{\prime}\left(t_{1}\right) \geq v^{\prime}\left(t_{1}\right)>0,
$$

but this contradicts the condition (3.20). This contradiction proves Theorem 3.2.

Note. - The equation (3.12) satisfies the restriction given in the Theorem, e.g. if $l_{1}(t) \equiv 0$ or if $l_{1}(t)=-c^{2} t^{-2}$ and $l_{2}(t)=(2 c-1) t^{-1}$, where $c \geq 0$.

\section{REFERENCES}

[1] P. Hartman and A. Wintner, on the non-increasing solutions of $y^{\prime \prime}=f\left(x, y, y^{\prime}\right)$, Amer. Journal of Math., vol. 73, N. 2 (1951), pp. 390.404.

[2] A. KNESER, Untersuchung und asymptotische Darstellung der Integrale gewisser Differen. tialgleichung bei grossen reellen Werthen des Arguments, I, Journal für die reine und angew. Math., vol 116 (1896), pp. 178.212.

[3] L. V. Kantorovic and G. P. AkrLov, Functional Analysis in Normed Spaces, Moscow, 1959 (Russian).

[4] G. Sansone. Ordinary Differential Equations, vol, 2 Moscow, 1954 (Russian). 